\title{
Development of analysis methods for ensuring the reliability of gas distribution systems in the problems of complex energy supply
}

\author{
Nikolay I. Ilkevich ${ }^{l}$, Tatyana.V. Dzyubina ${ }^{l, *}$, Zhanna.V. Kalinina ${ }^{l}$ \\ ${ }^{1}$ Melentiev Energy Systems Institute of Siberian branch of Russian Academy of Science (ESI SB \\ RAS), department of pipeline and hydraulic systems, Lermontov street 130, Irkutsk, Russia, 664033
}

\begin{abstract}
The paper deals with the issues devoted to reliability studies of gas distribution systems for complex energy supply of large industrialized areas. Given is a brief review of the reliability assessment methods, existing models for reliability assessment of the main pipelines, and their implementation. Special attention is paid to such reliability-specific properties as failure-free operation and maintainability of the elements constituting the gas distribution systems (linear sections and booster stations). These properties depend on the failure and recovery rates. Consideration is given to potentials of studying the impact of failure and recovery rates on integral indicators of gas distribution systems reliability. For that purpose mathematical models are proposed to be used for gas distribution systems reliability assessment using the analytical method of a 'loss - multiplication' scheme. Reliability assessment of a conventional gas distribution system was taken as an example.
\end{abstract}

\section{Introduction}

Analysis of natural gas consumption in Russian Federation during the latest several years [1] demonstrates gas consumption growth in large industrial complexes and in the residential sector (primarily by utilities and population) due to rapid construction of new residential agglomerations, cottage and summer cottage settlements. Demand for electric and heat energy grows accordingly.

Distributed power generation is currently a world-wide tendency aimed at meeting such a demand. Due to a number of advantages, in the nearest and remote perspective natural gas will become the main fuel used for centralized and distributed electric and heat energy production.

Therefore, large-scale gasification of big industrialized areas for the purpose of their energy supply necessitates development of centralized gas supply and gas distribution systems, and their expansion.

Total length of gas distribution systems increases accordingly, and configuration of gas pipeline schemes becomes more sophisticated. Pipes are manufactured using new materials, new thermal and hydro insulation technologies are used, processes of pipes corrosion and

* Corresponding author: tvleo@isem.irk.ru 
scaling are minimized, new highly maneuverable boosting and power equipment, and highquality valves are integrated.

All the above factors enhance the reliability of gas distribution systems while energy consumers pose more and more stringent requirements to their failure-free operation.

\section{Reliability problematics}

Reliability implies the ability of gas supply systems (GSS) to perform their functions in the specified scope under certain operating conditions, i.e., they shall supply gas to consumers and prevent circumstances hazardous for people and environment [2].

Gas distribution system (GDS) is a system consisting of gas pumping units of booster stations connected in series and in parallel for the purpose of gas supply for large industrialized areas.

A gas distribution system is a part of a sophisticated gas transportation system that includes a large number of different units, such as gas distribution stations, booster stations, and linear sections of pipelines, line booster stations for gas boosting, tie-lines of a multiline pipeline, and other equipment and facilities.

Linear sections and booster stations are the major contributors into GDS reliability and cost.

According to the mathematical apparatus used, the reliability assessment methods can be divided into analytical methods [3], and statistical modeling methods [4]. In terms of reflecting the object's properties they can be divided into methods considering separate random states of an object, and methods considering a random process of the object operation.

Interest to the problems of the main pipeline reliability arose in Russia in the mid-70s [5-7 et al.], Melentiev Energy Systems Institute, Siberian Branch of the Russian Academy of Sciences (MESI SB RAS) being among those interested in that subject [8].

A large number of papers have been devoted to this issue. Some studies use analytical methods of continuous-time Markovian chain theory both at the level of random events [5] and at the level of random processes $[6,8]$. There exist reliability assessment methods that take into account hydraulics [7]. Ref. [6] proposes a technique based on Monte-Carlo method using two models of a gas pipeline, namely, probabilistic and hydraulic ones, that are based on random events; Ref. [9] offers using the method of statistical tests for solving the gas pipeline reliability problems, but at a level of random events.

The most complete overview of techniques and mathematical models for assessing the gas pipeline reliability (including main pipelines and gas distribution systems) that were developed in Russia in 1980-1990 is given in the reference book [10].

Gas supply systems of the scale similar to Russian gas-supply systems are not common for other countries, and, therefore, they are less interested in modeling the reliability of GSS and its elements (booster stations and MG). But lately more and more studies have been devoted to gas pipeline reliability [11-14], the latest ones using the method of statistical tests. Mention can also be made of some standard software currently applied for assessment of gas supply reliability to consumers, e.g., Relex Reliability Studio 2007 [15] or STATISTICA that have been developed in the USA [16].

Workers of MESI SB RAS continue investigation of the main reliability problems of GDS and main pipelines [17]. Main pipeline reliability is analyzed using the analytical method at the level of random processes based on the 'loss - multiplication' scheme from the queueing theory $[18,19]$. The basic primary reliability information includes rate of elements' failure, i.e., individual parts of linear sections, gas pumping units, etc., $\lambda=1 / T_{p}$ and rates of their recovery $\mu=1 / T_{\theta}$, where $T_{p}$ is mean time to failure, and $T_{\beta}$ is mean 
recovery time. These indicators reflect such reliability properties as failure-free operation and maintainability. It should be noted that this approach was used by some other authors $[10,20]$ as well. Failure-free operation as a reliability property was prioritized. At the same time it is well-known that element's backup (redundancy) and proper maintenance of failed equipmnet are the main ways of enhancing the reliability of system's components.

To conclude, it should be mentioned that rather efficient methods with different degree of simplifications and assumptions have been developed for gas pipeline reliability assessment. However, all those studies do not pay due attention to the impact of failure and recovery rates on the integral reliability indicators of GDS, which makes this particular study relevant.

\section{Mathematical model for GDS reliability analysis}

This model gives the assessment only. The object of study is an aggregate of linear sections of pipelines and booster stations connected in parallel and in series [17].

A one-line diagram of gas-distribution system is specified, i.e., the number of lines of different diameter between a gas distribution station of the main pipeline and gas consumers of a large industrialized area. Some gas pipelines may have a booster station to balance pressure losses in the pipelines to remote consumers. For these pipelines that may consist of several lines, we specify the number of linear sections, the number of booster stations, and the number of installed and backup gas-pumping units (GPU). Backup units are assumed to be available at booster stations only. Linear part of GDS is not backed-up.

Reliability properties of GDS taken into account include failure-free operation and maintainability (specified are the failure and recovery rates of lines and gas pumping units). Flows of equipment failures and recovery (GPU and pipe runs) are assumed to be the simplest flows.

Only emergency shutdowns of equipmnet, current and preventive maintenances are taken account of.

In the general form the reliability assessment problem is stated as follows: integral indicators of GDS reliability are determined based on the scheme of GDS elements connection, type of backup, reliability of linear sections of pipelines and GPU that characterize the failure rate and maintainability.

GDS reliability analysis $[17,18]$ is made using the 'loss - multiplication' scheme. This analytical method that considers an object at a level of a random process is used for elements recovered after repair and implies availability of backups. It works well for reliability analysis of the systems of homogeneous elements [18]. GDS in this paper is considered as an aggregation of homogeneous and non-homogeneous systems; for making it homogeneous (in the latter case) the equivalency methods are used [21].

The 'loss - multiplication' scheme implies that every failed unit is taken from a reserve, and every repaired unit is forwarded to a reserve. Every failed component (a line or a unit) is assumed to be maintained by one maintenance team. If all the maintenance teams are engaged, the failed component is queued and waits for repair.

According to the 'loss - multiplication' process, probability of the fact that $0,1,23$, etc. elements of the system fail is computed using the iteration method [17].

This allows determination of the line sections and booster stations availability distribution series. Then distribution series are multiplied both for components connected in parallel and in series. In the former case the probability products would correspond to the sum of transfer capacities of the components involved, and in the latter case - to discrete transfer capacity related to its least value in one of the distribution series.

The result of those computations is the final series of GDS availability probability distribution as a whole that is further used for determination of integral reliability indicators 
[17], i.e., statistical expectation of GDS transfer capacity $M(Q)$, and its reliability factor $\left(K_{\mathrm{r}}\right)$.

\section{An example of GDS reliability indicators computation}

To exemplify the proposed approach, consideration was given to a conventional gas pipeline system consisting of two lines working in parallel; an equivalent GDS scheme is given in Fig. 1. A booster station includes five gas pumping units working in parallel (four in operation and one backup).

Integral reliability indicators are affected by the number of maintenance teams; in this particular case this factor was disregarded. Each linear section and booster station is assumed to be assigned one maintenance team.

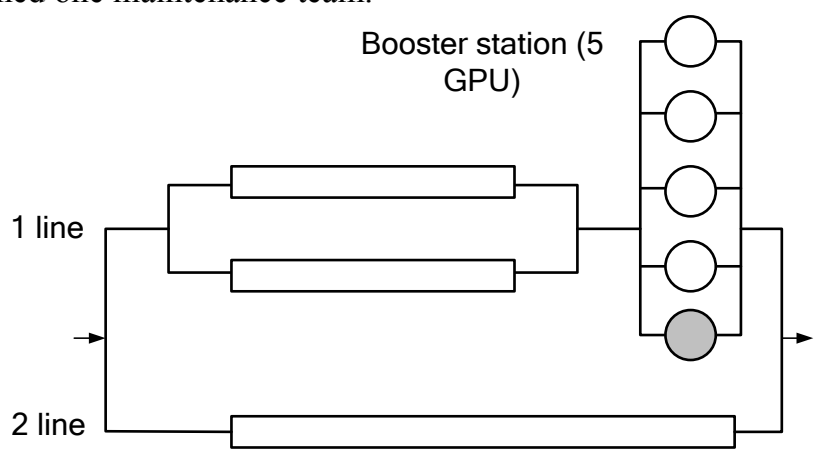

Fig. 1. An equivalent scheme of a conventional gas distribution system

This gas distribution system feeds gas from GDS of the main pipeline to industrial consumers of a large industrialized area. Its transfer capacity is $Q_{\mathrm{H}}=0,4 \mathrm{bcm} / \mathrm{yr}$; its length is $250 \mathrm{~km}$. Input conventional indicators for a linear section are as follows: $\lambda=0,2 \cdot 10^{-3} 1 / \mathrm{yr}$ $\mathrm{km}, \mu=108,041 / \mathrm{y}$ (one line), $\lambda=0,63 \cdot 10^{-3} 1 / \mathrm{yr} \mathrm{km}, \mu=171,441 / \mathrm{yr}$ (two lines); for a gas pumping unit: $\lambda=3,65 \cdot 1 / \mathrm{yr}$ per unit, $\mu=90,881 / \mathrm{yr}$.

Mathematical models for GDS reliability analysis allowed computation of probability distribution series of conventional GDS availability. Statistical expectation of its transfer capacity equals $M(Q)=0,392 \mathrm{bcm} / \mathrm{yr}$, reliability factor is $K_{\mathrm{H}}=0,392 / 0,4=0,98$.

Impact of failure and recovery rates on GDS integral reliability indicators was also assessed. Studies have shown that if failure rate of pipelines and GPU is $10 \%$ higher than the basic option (under constant rate of their recovery), the reliability factor is $K_{\mathrm{H}}=0,972$; if recovery rate of pipelines and GPU is $10 \%$ lower than the basic option (under constant rate of their failures), the reliability factor equals $K_{\mathrm{H}}=0,984$.

Thus, it is obvious that with the change in input reliability indicators of GDS components by the same value, their impact on the change in the integral GDS reliability indicators is different. Such studies could be useful for investigating the impact of input reliability indicators of different equipment on integral reliability indicators of sophisticated equipment.

\section{Conclusion}

An ever-increasing role of natural gas in the integrated energy supply of large industrialized areas stipulates relevancy of studies on enhancement of methods for reliability analysis of gas distribution systems. The proposed mathematical model for GDS reliability analysis 
allows study of the impact of failure and recovery rates of the elements that are responsible for failure-free operation and maintainability of gas transportation systems on their integral reliability indicators.

The research was carried out within the project III.17.4.3 of the Fundamental research program of $S B$ RAS (AAAA-A17-117030310437-4)

\section{References}

1. http://www.gazprom.ru/investors/reports/2013/

2. Ed. by N.I. Voropai, Reliability of energy systems. Reference book of terms (2007)

3. E.S. Vetncel, The theory of probability (2001)

4. N.P. Buslenko, U.A. Shneider, Method of statistical testing (Monte-Carlo method) (1969)

5. A.A. Afanasiev, Yu.I. Kifer, A.M. Podberezkin, A.L. Raikin, Reliability analysis methods for gas transportation and gas producing companies (1975)

6. E.P. Stavrovsky, M.G. Sukharev, A.M. Karasevich, Methods for assessment of the main gas pipelines reliability (1982)

7. E.L. Volsky, A.I. Garlyauskas, S.V. Gerchkov, Reliability and optimum backup of gas fields and main pipelines (1980)

8. N.I. Ilkevich, Methodological issues of reliability studies of the large energy systems, 1, 50 (1974)

9. E.P. Akoev, Gas transport and storage, 11, 17 (1978).

10. Edited by M.G. Sukharev, Reliability of gas and oil supply systems (1994)

11. Convention on the transboundary effects of industrial accidents. Safety Guidelines and Good Practices for Pipelines (2008)

12. $\mathrm{Wu} \mathrm{Xia}, \mathrm{Li}$ Changjun, $\mathrm{He}$ Yufa, Jia Wenlong. Mathematical Problems in Engineering Volume, 18 (2018)

13. M. Witek, Pipeline Technology Journal, 3, 16 (2018)

14. M. Witek, Engineering Failure Analysis, 70, 255 (2016)

15. Relex Curriculum Guide (2007)

16. Sá. Joaquim. Applied Statistics Using Spss, Statistica, Matlab and R (2007)

17. N.I Ilkevich, T.V. Dzyubina, Zh. V. Kalinina, Multi-level modeling of gas supply systems development (2014)

18. O.A. Novikov, S.I. Petukhov, Applied issues of Queueing Theory (1969)

19. Dr. János Sztrik, Basic Queueing Theory, University of Debrecen, Faculty of Informatics (2012)

20. E. A. van Doorn, Shell Polynomials and Dual Birth-Death Processes, 12, 15 (2016)

21. M.A. Dubitsky, Yu.N. Redunko, M.B. Cheltsov, Selection and use of generating power reserve in the electric power systems (2015) 